\title{
Are the Clinical outcomes of Neonates and Infants Under 2 Months Old with Urinary Tract Infections Similar to those in Infants 2 to 12 Months Old?
}

\author{
Jee Hoo Lee, M.D., \\ Hyunwook Lim, M.D., \\ Kyungju Kim, M.D., \\ Hyung Eun Yim, M.D., Ph.D., \\ Kee Hwan Yoo, M.D., Ph.D. \\ Department of Pediatrics, College \\ of Medicine, Korea University, Seoul, \\ Korea
}

\section{Corresponding author: \\ Kee Hwan Yoo, M.D., Ph.D. \\ Department of Pediatrics, College of Medicine, Korea University, Seoul, Korea \\ Tel: +82-2-2626-1229 \\ Fax: +82-2-2626-1249 \\ E-mail: guroped@korea.ac.kr}

Received: 18 August 2015

Revised: 7 September 2015

Accepted: 13 October 2015

\begin{abstract}
Purpose: Although the American Academy of Pediatrics provides clinical guidelines for urinary tract infection (UTI) infants, guidelines are not appropriate for neonates and infants less than 2 months of age due to insufficient data. The aim of this study was to evaluate the characteristics of neonates and young infants less than 2 months old (group 1) with UTI compared to older infants from 2 to 12 months old (group 2).

Methods: We reviewed UTI patients aged 0 to 12 months admitted to the pediatric department in the last 5 years. Clinical characteristics such as age, sex, fever duration, recurrence, progression to acute pyelonephritis (APN), malformations like hydronephrosis and vesicoureteral reflux (VUR), and laboratory results were compared between group 1 and group 2 .

Results: 615 patients were included in this study. Group 1 had 94 cases and group 2 had 521 cases. Escherichia coli was the most commonly isolated pathogen in urine cultures. Fever duration was shorter in group 1 (vs.) 2 ( $1.91 \pm 1.43$ days vs. $3.42 \pm 2.40$ days, $P<0.05$ ). As compared to group 2 , group 1 had a higher proportion of patients with antenatal hydronephrosis and hydronephrosis found after admission ( $10.6 \%$ vs. $3.6 \%$ and $75.5 \%$ vs. $55.9 \%, \mathrm{P}<0.05)$. There were differences between two groups in white blood cell (WBC) count (Group 1: 13,694 $\pm 5,315 / \mathrm{hL}$, Group 2: $15,271 \pm 6,130 / \mu \mathrm{L}, P<0.05$ ) and C-reactive protein (Group 1: 32.02 \pm 35.17 $\mathrm{mg} / \mathrm{L}$, Group 2: $46.51 \pm 46.63 \mathrm{mg} / \mathrm{L}, P<0.05)$.

Conclusion: Compared to older infants, UTI in neonates and young infants shows milder clinical manifestations except higher rates of hydronephrosis but outcome is alike.
\end{abstract}

Key words: Urinary tract infection, Young infant, Hydronephrosis

\section{Introduction}

Urinary tract infection (UTI) is the most common bacterial infection in febrile infants, especially in neonates and young infants ${ }^{1,2)}$. The overall prevalence of UTIs through childhood age is about $1 \%$ in boys and $1-3 \%$ in girls ${ }^{3}$. The prevalence in neonates and young infants is higher: $12 \%$ in males and 5\% in females $^{2)}$. It is important to consider UTIs in febrile infants because of its potential complications, like renal scarring, which could lead to renal tissue damage before kidney development is completed. In addition, hypertension 
and end-stage renal disease can occur when treatment is delayed $^{4,5)}$.

However, it is challenging to diagnose UTIs in neonates and infants because of its vague symptoms, which are fever, lethargy, poor feeding, vomiting, and abdominal pain. Moreover, collecting urine samples for analysis and culture from this age group is difficult and mostly needs to be performed by invasive methods such as catheterization or suprapubic aspiration ${ }^{6,7}$. The American Academy of Pediatrics (AAP) and National Institute for Health and Care Excellence (NICE) guidelines recommend obtaining a proper urine specimen from febrile infants for analysis and culture within 24 hours if possible. The urine specimen should be obtained by catheterization or suprapubic aspiration, and should be obtained before administration of antibiotics ${ }^{6,7)}$.

There are several studies about UTIs in neonates and young infants that examine the characteristics of UTIs in young infants ${ }^{8-10)}$, including laboratory results ${ }^{11)}$, clinical courses $^{12)}$, the association with bacteremia or bacterial meningitis ${ }^{12,13)}$, and factors related to the recurrence of febrile UTIs ${ }^{8}$. However, it is hard to find a comparative study examining differences between young infants (less than two months of age) and older infants.

The $\mathrm{AAP}^{6)}$ and $\mathrm{NICE}^{7)}$ provide clinical guidelines for clinicians treating febrile pediatric patients about when to suspect patients of having a UTI, when to perform urine analysis with or without culture, and the management of UTIs, including imaging studies. AAP guidelines are mainly based on evidence derived from studies where the subjects are infants and children aged between 2 and 24 months. Therefore, the guidelines are not appropriate to apply to neonates and infants younger than 2 months of age because of insufficient data ${ }^{6}$. The purpose of this study was to evaluate clinical characteristics, laboratory results, and imaging studies of neonates and young infants less than 2 months old compared with those of older infants between the ages of 2 and 12 months.

\section{Materials and Methods}

We collected data from all pediatric patients admitted to the Korea University Guro Hospital from January 2010 to June 2015 with the diagnosis of "urinary tract infection, site not specified" (N39.0), "neonatal urinary tract infection" (P39.3), "acute pyelonephritis" (N10C), or "pyelonephritis NOS" (N12C). These diagnoses are defined in the Korean Classification of Diseases (KCD)-6. Among 1,084 patients, 745 patients were younger than 12 months of age. The inclusion criteria were as follows: 1) patients with aforementioned diagnoses accompanying fever. Fever was defined as a body temperature of at least $38.0^{\circ} \mathrm{C}$, as measured by an infrared tympanic membrane thermometer ${ }^{6}$. 2) patients whose urine culture was performed by catheterization or suprapubic aspiration and 3) patients whose urine culture was positive. Urine culture was considered positive if it showed $>50,000$ colony forming units (CFUs) of a single pathogen or $>10,000$ CFUs of a single pathogen when patients were symptomatic ${ }^{3,14)}$. As a result, 615 patients were enrolled and divided into two groups: group 1 and group 2 . Group 1 included neonates and infants younger than 2 months of age and group 2 included older infants from 2 to 12 months of age.

Patients' medical records about clinical manifestation, laboratory results, and imaging studies were reviewed retrospectively. Clinical manifestations were age, sex, admission and discharge dates, causative organisms, fever duration before and during hospitalization, previous UTI history, number of recurrences, and accompanying malformations other than hydronephrosis or vesicoureteral reflux (VUR).

The laboratory tests were conducted to determine peripheral white blood cell (WBC) count $(/ \mu \mathrm{L})$, blood urea nitrogen (BUN) (mg/dL) level, serum creatinine (mg/dL) level, and serum C-reactive protein (CRP) (mg/L) level in blood, and detect pyuria, hematuria, proteinuria, and nitrite in urine samples. Pyuria was defined as +1 and above in a dipstick test or more than 5 WBCs /high power field (HPF) by microscopic examination. Hematuria was defined as +1 and above in a dipstick test or more than 5 red blood cell/HPF by microscopic examination. Proteinuria and nitrite were also defined as +1 and above in a dipstick test.

Imaging studies included abdominal ultrasonography, Technetium (Tc)-99m dimercaptosuccinic acid (DMSA) renal scan, and voiding cystourethrography (VCUG). Abdominal ultrasonography was performed in 611 
Table 1. Clinical characteristics of UTI in neonates/young infants (group 1) and older infants (group 2)

\begin{tabular}{|c|c|c|c|}
\hline & Group 1 & Group 2 & $P$-value \\
\hline Total patients & 94 & 521 & \\
\hline Male to female ratio & $3.95: 1$ & $2.30: 1$ & $N S^{*}$ \\
\hline Age (months) & $1.3 \pm 0.6$ & $5.3 \pm 2.6$ & $<0.001$ \\
\hline Previous UTI history & $0(0 \%)$ & $13(2.5 \%)$ & $N S^{*}$ \\
\hline Fever before admission (days) & $1.06 \pm 0.85$ & $2.27 \pm 2.14$ & $<0.001$ \\
\hline Fever after admission (days) & $0.85 \pm 1.07$ & $1.16 \pm 1.25$ & $<0.05$ \\
\hline Total fever duration (days) & $1.91 \pm 1.43$ & $3.42 \pm 2.40$ & $<0.001$ \\
\hline Admission duration (days) & $9.33 \pm 2.64$ & $8.88 \pm 1.86$ & $N S^{*}$ \\
\hline Recurrence & $6(6.40 \%)$ & $37(7.10 \%)$ & $N S^{*}$ \\
\hline Accompanied malformations & $5(5.30 \%)$ & 17 (3.30\%) & $N S^{*}$ \\
\hline
\end{tabular}

NS*: Non-significant

patients, DMSA renal scan was done in 595 patients, and 431 patients underwent VCUG.

Hydronephrosis was graded as described in the ultrasound grading system of hydronephrosis by the Society for Fetal Urology (SFU) ${ }^{15)}$. VUR was graded from grade I to $\mathrm{V}$, as referred to in the international system of radiographic grading of vesicoureteric reflux ${ }^{16)}$.

Data collected from the two groups were compared and analyzed statistically with IBM SPSS Statistics version 21.0.0.0 (SPSS Inc., Chicago, IL, USA). The chi-square test was used to compare categorical variables and the Fisher's exact test was used when more than $20 \%$ of cells had an expected frequency of five or less. Continuous or ordinal variables were analyzed by an independent sample t-test or Mann-Whitney U test. A P-value was considered statistically significant when it was smaller than 0.05 .

\section{Results}

Among the 615 patients, 94 patients were in group 1 and 521 patients were in group 2 . The male to female ratio was 3.95:1 in group 1 and 2.30:1 in group 2. The mean age of group 1 was $1.3 \pm 0.6$ months and that of group 2 was $5.3 \pm 2.6$ months. None of the patients in group 1 had a previous history of UTI, but 13 patients (2.5\%) in group 2 had a previous UTI history.

\section{Clinical manifestations}

Clinical manifestations are shown in Table 1. Fever duration before hospitalization was an average of $1.06 \pm 0.85$ days in group 1 and $2.27 \pm 2.14$ days in group $2(P<0.001)$. Fever duration after hospitalization was $0.85 \pm 1.07$ days and $1.16 \pm 1.25$ days $(P<0.05)$ and total fever duration was $1.91 \pm 1.43$ days and $3.42 \pm 2.40$ days in group 1 and group 2, respectively $(P<0.001)$. Recurrence rates after discharge in the two groups were not statistically different. Accompanied malformations other than hydronephrosis and VUR were found in 5 cases (5.3\%) in group 1 and 17 cases (3.3\%) in group 2. The malformations were duplication of the kidney, kidney hypoplasia, megaloureter, periureteral or bladder diverticulum, multicystic dysplastic kidney, ectopic location of kidney, hypospadias, and concealed penis.

\section{Imaging studies}

Results of abdominal ultrasonography are shown in Table 2 which is comparing proportion and characteristics of hydronephrosis between two groups. In group 1, 10 of 94 patients (10.6\%) had congenital hydronephrosis, while 19 of 521 patients (3.6\%) in group 2 had congenital hydronephrosis $(P<0.01)$. Hydronephrosis detected by ultrasonography during hospitalization was more frequent in group 1 (75.5\%) than group $2(55.9 \%)(P<0.001)$. Bilateral hydronephrosis was also more frequent in group 1 (24.5\%) than group $2(10.4 \%)(P<0.001)$. Among the 440 kidneys that showed hydronephrosis, the grade of hydronephrosis was compared between the two groups. In group 1, 56.4\% of the kidneys were diagnosed as high-grade (grade II to IV) whereas $44.2 \%$ of the kidneys in group 2 were diagnosed as high-grade $(P<0.05)$. The average grade of hydronephrosis in group $1(1.78 \pm 0.84)$ was higher than in group 2 $(1.48 \pm 0.58)(P<0.05)$. 
Table 2. Hydronephrosis in neonates/young infants (group1) and older infants (group 2)

\begin{tabular}{lccc}
\hline & Group 1 & Group 2 & $P$-value \\
\hline Congenital hydronephrosis & $10(10.60 \%)$ & $19(3.60 \%)$ & $<0.05$ \\
Hydronephrosis & $71(75.50 \%)$ & $289(55.90 \%)$ & $<0.001$ \\
Bilateral hydronephrosis & $23(24.50 \%)$ & $54(10.40 \%)$ & $<0.001$ \\
Gr * $^{*}$ Gr II IV $^{*}$ & $41(43.60 \%)$ & $193(55.80 \%)$ & $<0.05$ \\
Grade of hydronephrosis & $53(56.40 \%)$ & $153(44.20 \%)$ & $<0.05$ \\
\hline
\end{tabular}

*Number of the kidneys affected by hydronephrosis.

Table 3. Comparison of laboratory results between neonates/young infants (group1) and older infants (group 2)

\begin{tabular}{|c|c|c|c|}
\hline & Group 1 & Group 2 & $P$-value \\
\hline White blood cell count $(/ \mu \mathrm{L})$ & $13,694 \pm 5,315$ & $15,271 \pm 6,130$ & $<0.05$ \\
\hline Neutrophil (\%) & $46.93 \pm 14.92$ & $44.47 \pm 17.36$ & $N S^{*}$ \\
\hline Absolute neutrophil count $(/ \mu \mathrm{L})$ & $6,695 \pm 4,245$ & $7,285 \pm 4,728$ & $N S^{*}$ \\
\hline C-reactive protein $(/ \mu \mathrm{L})$ & $32.02 \pm 35.17$ & $46.51 \pm 46.63$ & $<0.05$ \\
\hline Erythrocyte sedimentation rate ( $\mathrm{mm} / \mathrm{hr})$ & $34.09 \pm 29.81$ & $38.87 \pm 28.02$ & $N S^{*}$ \\
\hline Pyuria (dipstick) ${ }^{\dagger}$ & 65 (69.10\%) & 402 (77.20\%) & NS* \\
\hline Pyuria (micro) ${ }^{\dagger}$ & $65(69.10 \%)$ & $409(78.50 \%)$ & $<0.05$ \\
\hline Nitrite $^{+}$ & $27(28.70 \%)$ & $138(26.50 \%)$ & $N S^{*}$ \\
\hline 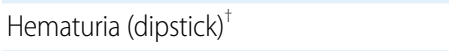 & $61(64.90 \%)$ & $277(53.20 \%)$ & $<0.05$ \\
\hline Hematuria $\left(\right.$ micro) $^{\dagger}$ & $49(52.10 \%)$ & 223 (42.80\%) & $N S^{*}$ \\
\hline Proteinuria $^{\dagger}$ & $43(45.70 \%)$ & $230(44.10 \%)$ & $\mathrm{NS}^{*}$ \\
\hline
\end{tabular}

NS*: Non-significant

${ }^{\dagger}$ Urinalysis results are compared by the number and percentage of patients who are positive in each test between the two groups.

A DMSA renal scan was done in 595 (96.7\%) of the total 615 patients, and 179 (15.0\%) out of 1,190 kidneys showed a cortical defect. The number of kidneys that had cortical defects in group 1 (11.3\%) and group 2 (15.7\%) showed no statistically significant difference. Bilaterality of APN in both groups was also not statistically different.

VCUG was performed on 431 (70.1\%) patients; only 69 kidneys (8.0\%) had VUR among the total 862 kidneys that were evaluated. VUR was present in 14 (9.7\%) out of 144 kidneys of group 1 and 55 (7.7\%) out of 718 kidneys of group 2; the difference between the two groups was not significant. Grade and bilaterality of VUR also showed no difference between the two groups.

\section{Laboratory tests}

Laboratory test results are shown in Table 3. Escherichia coli was the most commonly isolated pathogen, and was present in $80.9 \%$ of patients in group 1 and $81.6 \%$ in group 2. The second most common pathogen was Klebsiella species (5.3\% in group 1 and 9.8\% in group 2), followed by En- terobacter species (1.1\% in group 1 and $3.1 \%$ in group 2), and Enterococcus species (5.3\% in group 1 and $1.9 \%$ in group 2). The difference in the proportion of each pathogen between the two groups was not statistically significant.

Peripheral WBC count and serum CRP levels were significantly different in the two groups. Peripheral WBC count (group 1, 13,694 $\pm 5,315 / \mu \mathrm{L}$ and group 2, 15,271 $\pm 6,130 / \mu \mathrm{L}$, $P<0.05$ ) and serum CRP (group 1, 32.02 $\pm 35.17 \mathrm{mg} / \mathrm{L}$ and group $2,46.51 \pm 46.63 \mathrm{mg} / \mathrm{L}, P<0.05)$ were lower in group 1 than group 2.

Pyuria was more frequently seen in group $2(78.5 \%)$ than in group $1(69.1 \%, P<0.05)$. Conversely, hematuria was more common in group 1 (64.9\%) compared to group $2(53.2 \%, P<0.05)$. Other laboratory results were not statistically significantly different between the two groups.

\section{Discussion}

UTIs must be considered a fever focus in febrile infants 
because they are the most common bacterial infection in young febrile infants, and, if not treated, can result in permanent renal tissue damage ${ }^{1,2,45}$. The AAP has provided general guidelines for managing febrile infants with UTI, but the guidelines have limited applicability to infants within the age of 2-24 months, and there is insufficient data with regard to UTI in neonates and infants younger than 2 months old ${ }^{6}$. Therefore, it is necessary to collect and compare clinical characteristics of UTI in neonates and young infants with older infants aged 2 to 24 months in order to reach a consensus on the management of neonates and young infants with febrile UTI. The aim of this study was to compare clinical characteristics, results of imaging studies, and laboratory tests between younger and older infant age groups.

In this study, fever duration was shorter in the younger age group than the older age group. In a study by Eun et al. ${ }^{13)}$, they noted that the mean duration to defervescence after antibiotic administration was $1.7 \pm 1.1$ (range, 0-6) days in febrile UTI patients aged younger than 3 months old, which is longer than the results of our study. Similar to our study, Bachur ${ }^{17)}$ showed that mean fever duration was shorter in younger patients compared to older UTI patients. Variation of fever duration among these studies might be due to different study populations and may be affected by differences in the pre-hospital duration of fever, which these authors did not report.

Park et al. ${ }^{8)}$ reported hydronephrosis found by ultrasonography in $66.7 \%$ of UTI patients less than two months old. Bonadio et al. ${ }^{10)}$ also noted hydronephrosis was present in $47.4 \%$ of infants younger than 30 days old with UTIs. Our study results showed that hydronephrosis was detected in $75.5 \%$ of patients in the younger age group during hospitalization and in $55.9 \%$ of patients in the older age group. However, Coe et al. ${ }^{18)}$ and Kanellopoulos et al. ${ }^{19)}$ reported different hydronephrosis detection rates in UTI patients than the studies previously cited, $23.1 \%$ and $7.7 \%$ respectively. These differences might be due to the different age groups enrolled in each study; the latter two studies included patients older than 12 months of age. Another cause might be inter-examiner variation, which can influence diagnosis and grading of hydronephrosis.

As shown in our study, the tendency toward a higher prevalence of antenatal hydronephrosis and hydrone- phrosis detected during hospitalization in the younger age group was present throughout several studies. This could result from the characteristics of antenatal hydronephrosis, whose most common cause is self-limited collecting system dilation. It has been reported that transient dilation is the cause of antenatal hydronephrosis in up to $55 \%$ of cases $^{20}$. Coelho et al. ${ }^{21)}$ reported idiopathic dilatation as a postnatal diagnosis in $67 \%$ of infants with isolated antenatal mild hydronephrosis. In addition, Alconcher et al. $^{22)}$ also noted that $80 \%$ of antenatally detected hydronephrotic kidneys showed total resolution during the first year of life.

Hydronephrosis is considered an important factor when considering UTI recurrence. Park et al. ${ }^{8)}$ reported a 50\% recurrence rate in patients with hydronephrosis younger than 2 months of age and $100 \%$ of patients with recurrent UTI had hydronephrosis. However, in this study, there was no difference in the recurrence rate, even with the higher incidence of hydronephrosis in the younger age group.

A DMSA scan is one of the most sensitive and useful ways to diagnose acute pyelonephritis and detect renal scarring after $\mathrm{UTI}^{23,24)}$. Cortical defects were noted in $27.4 \%$ of patients and $15.0 \%$ of kidneys in our study. This result is similar to other studies. For example, Park et al. ${ }^{8)}$ reported cortical defects in $21.8 \%$ of patients, Kang et al. ${ }^{25)}$ reported $37.1 \%$, and Camacho et al. ${ }^{26)}$ reported cortical defects in $26 \%$ of patients. There was no difference in the incidence of cortical defects between the younger and older age group in this study. This result was consistent with another study by Kanellopoulos et al. ${ }^{19)}$, which compared neonates, infants, and young children less than 24 months of age. The results of their study showed no differences between age groups with regard to the incidence of pyelonephritis and urinary tract abnormalities, other than reflux or pyelonephritis.

In our study, VUR was noted in $12.5 \%$ of patients and $8.0 \%$ of kidneys. This incidence was slightly lower than seen in other studies. Studies by Park et al. ${ }^{8}$, Kang et al. ${ }^{25}$ and Coe et al. ${ }^{18)}$ noted a VUR incidence of $20.4 \%, 27.7 \%$ and 29\%, respectively. However, the incidence of VUR between the younger and older age groups was not different in this study. Also, Kanellopoulos et al. ${ }^{19)}$ reported no difference in the incidence of VUR between neonates, infants, and young children. 
In this study, the WBC count was lower in the younger age group compared to the older age group. CRP was also lower in the younger age group than the older age group. Koufadaki et al. ${ }^{27)}$ stated in their study that patients with a $\mathrm{WBC}$ of $\geq 18,000 / \mu \mathrm{L}$ or $\mathrm{CRP} \geq 50 \mathrm{mg} / \mathrm{L}$ were 2.44 times and 2.67 times more likely, respectively, to develop moderate to severe lesions in a DMSA renal scan. However, the mean WBC count and CRP in our study were not high enough in either group to meet the cut-off value that they proposed. In fact, there were no statistically significant differences in the prevalence of cortical defects in the DMSA renal scans between two groups.

In conclusion, fever resolved more quickly in the younger age group than the older age group in response to antibiotic treatment. The peripheral WBC count and CRP levels were also lower in the younger age group. Other clinical manifestations investigated in this study, such as kinds of causative pathogens, prevalence of cortical defects in DMSA renal scan, VUR, accompanied malformations, and recurrence rates were not significantly different between the two groups. Only hydronephrosis was more common in the younger age group.

\section{References}

1. Mårild S, Jodal U. Incidence rate of first-time symptomatic urinary tract infection in children under 6 years of age. Acta Paediatr 1998;87(5):549-52.

2. Shaikh $\mathrm{N}$, Morone NE, Bost JE, Farrell MH. Prevalence of urinary tract infection in childhood: a meta-analysis. Pediatr Infect Dis J 2008;27(4):302-8.

3. Elder JS. Urinary Tract Infections. In: Kliegman RM, Stanton BF, St Geme JW, Schor NF, editors. Nelson textbook of pediatrics. 20th ed. Philadelphia: Saunders Elsevier, 2015: 2556-62.

4. Lee SJ. Guidelines for childhood urinary tract infection. Korean J Pediatr 2009;52(9):976-83.

5. Roberts KB, Akintemi OB. The epidemiology and clinical presentation of urinary tract infections in children younger than 2 years of age. Pediatr Ann 1999;28(10):644-9.

6. Roberts KB. Urinary tract infection: clinical practice guideline for the diagnosis and management of the initial UTI in febrile infants and children 2 to 24 months. Pediatrics 2011;128(3):595610.

7. National Institute for Health and Care Excellence. Urinary tract infection in children: Diagnosis, treatment and long-term management. Clinical Guideline no. 54, 2007. Available from:
http://www.nice.org.uk/guidance/CG54

8. Park KH, Lee EH, Kim MK, Lee JH, Choi BM, Yoo KH, et al. Febrile Urinary Tract Infection in Infants Less than Two Months of Age : Characteristics and Factors Related to the Recurrence.J Jorean Soc Neonatol 2012;19(2):91-7.

9. Ismaili K, Lolin K, Damry N, Alexander M, Lepage P, Hall M. Febrile urinary tract infections in 0 - to 3-month-old infants: a prospective follow-up study. J Pediatr 2011;158(1):91-4.

10. Bonadio W, Maida G. Urinary tract infection in outpatient febrile infants younger than 30 days of age: a 10 -year evaluation. Pediatr Infect Dis J 2014;33(4):342-4.

11. Lin DS, Huang SH, Lin CC, Tung YC, Huang TT, Chiu NC, et al. Urinary tract infection in febrile infants younger than eight weeks of age. Pediatrics 2000;105(2):e20.

12. Dayan PS, Hanson E, Bennett JE, Langsam D, Miller SZ. Clinical course of urinary tract infections in infants younger than 60 days of age. Pediatr Emerg Care 2004;20(2):85-8.

13. Eun BW, Chung YM, Kang HG, Ha IS, Cheong HI, Lee HJ, et al. Urinary tract infections in febrile infants under three months of age. Korean J Pediatr 2003;46(3):265-70.

14. Ahn HS. Hong's Textbook of Pediatrics. 10th ed. Seoul, Mirae \& Co., 2012: 910-5.

15. Fernbach S, Maizels M, Conway J. Ultrasound grading of hydronephrosis: introduction to the system used by the Society for Fetal Urology. Pediatr Radiol 1993;23(6):478-80.

16. Lebowitz R, Olbing H, Parkkulainen K, Smellie J, TamminenMöbius T. International system of radiographic grading of vesicoureteric reflux. Pediatr Radiol 1985;15(2):105-9.

17. Bachur R. Nonresponders: prolonged fever among infants with urinary tract infections. Pediatrics 2000;105(5):e59.

18. Coe HJ, Kim TH, Cho H, Kim JH. Comparison of the Clinical and Radiologic Characteristics between Different Age Groups with First Febrile UTI Under 2 Years of Age. Child Kidney Dis;11(2):22938.

19. Kanellopoulos TA, Salakos C, Spiliopoulou I, Ellina A, Nikolakopoulou NM, Papanastasiou DA. First urinary tract infection in neonates, infants and young children: a comparative study. Pediatr Nephrol 2006;21(8):1131-7.

20. Timberlake MD, Herndon CD. Mild to moderate postnatal hydronephrosis--grading systems and management. Nat Rev Urol 2013;10(11):649-56.

21. Coelho GM, Bouzada MC, Pereira AK, Figueiredo BF, Leite MR, Oliveira DS, et al. Outcome of isolated antenatal hydronephrosis: a prospective cohort study. Pediatr Nephrol 2007;22(10):1727-34.

22. Alconcher LF, Tombesi MM. Natural history of bilateral mild isolated antenatal hydronephrosis conservatively managed. Pediatr Nephrol 2012;27(7):1119-23.

23. Jakobsson B, Svensson L. Transient pyelonephritic changes on 99mTechnetium-dimercaptosuccinic acid scan for at least five months after infection. Acta Paediatr 1997;86(8):803-7.

24. Verber IG, Strudley MR, Meller ST. $99 \mathrm{mTc}$ dimercaptosuccinic 
acid (DMSA) scan as first investigation of urinary tract infection. Arch Dis Child 1988;63(11):1320-5.

25. Kang MJ, Shin HK, Yim HE, Je BK, Eun SH, Choi BM, et al. Urinary tract infections in infants under six months of age. Korean J Pediatr 2006:49(3):278-86.

26. Camacho V, Estorch M, Fraga G, Mena E, Fuertes J, Hernandez MA, et al. DMSA study performed during febrile urinary tract infection: a predictor of patient outcome? Eur J Nucl Med Mol Imaging 2004;31(6):862-6.

27. Koufadaki AM, Karavanaki KA, Soldatou A, Tsentidis C, Sourani MP, Sdogou T, et al. Clinical and laboratory indices of severe renal lesions in children with febrile urinary tract infection. Acta Paediatr 2014;103(9):e404-9. 